\title{
Solicitude em visita domiciliar de enfermeiras no cuidado pré- natal de alto risco: relato de experiência ${ }^{a}$
}

\author{
Solicitude in home visit of nurses in high-risk prenatal care: an experience report
} Solicitud en visita domiciliara de enfermeras en la atencíon prenatale de alto riesgo: relato de experiencia

Bruna Felisberto de Souza ${ }^{1}$ (1)

Bruna de Souza Lima Marski ${ }^{1}$ (1)

Maria Aparecida Bonelli ${ }^{1}$ (D)

Mariana Torreglosa Ruiz ${ }^{2}$ (1)

Monika Wernet ${ }^{1}$ (D)

1. Universidade Federal de São Carlos,

Programa de Pós-Graduação em Enfermagem.

São Carlos, SP, Brasil.

2. Universidade Federal do Triângulo Mineiro,

Programa de Pós-Graduação em Atenção à

Saúde. Uberaba, MG, Brasil.
Autor correspondente:

Bruna Felisberto de Souza.

E-mail: brunaf.sc@hotmail.com

Recebido em 20/08/2021.

Aprovado em 21/12/2021.

DOI:https://doi.org/10.1590/2177-9465-EAN-2021-0328

\section{RESUMO}

Objetivos: relatar a experiência de produção de cuidado no pré-natal de alto risco, por meio de visitas domiciliares estruturadas na solicitude. Método: estudo descritivo, na modalidade relato de experiência, pautado na vivência de enfermeiras em desenvolver visitas domiciliares, sob o referencial da solicitude para 17 mulheres com gravidez de alto risco. Resultados: as visitas domiciliares possibilitaram às enfermeiras a valoração dos aspectos subjetivos do cuidado, com apreensão de necessidades das mulheres e compreensão da gravidez na vida de cada uma delas, aspectos determinantes à atenção pré-natal integral. Promoveu cuidado singular e contraposição às insuficiências e negativas experienciadas na atenção pré-natal recebida, ancorada no modelo biomédico. Conclusão e implicações para a prática: a visita domiciliar por enfermeiras, sob os auspícios da solicitude revelou-se como tecnologia de atenção pré-natal de alto risco que produz acolhimento de necessidades singulares, assim como conduz a reconstrução do cuidado no contexto da gravidez de alto risco.

Palavras-chave: Cuidado Pré-Natal; Enfermeiras; Gravidez de Alto Risco; Integralidade em Saúde; Visita domiciliar.

\begin{abstract}
Objectives: to report the experience of producing high-risk prenatal care through home visits structured on request. Method: this is a descriptive study in the experience report modality, based on the experience of nurses in developing home visits under the solicitude framework for 17 women with high-risk pregnancies. Results: home visits allowed nurses to value the subjective aspects of care, with apprehension of women's needs and understanding of pregnancy in the lives of each one of them, determining aspects of comprehensive prenatal care. It promoted unique care and opposed to the insufficiencies and negatives experienced in the prenatal care received, anchored in the biomedical model. Conclusion and implications for practice: home visit by nurses under the auspices of solicitude has revealed itself as a high-risk prenatal care technology that produces reception of unique needs, as well as leads to care reconstruction in the context of high-risk pregnancy.
\end{abstract}

Keywords: Prenatal Care; Nurses; Pregnancy, High-Risk; Integrality in Health; House Calls.

\section{Resumen}

Objetivo: relatar la experiencia de producción del cuidado en la atención prenatal de alto riesgo a través de visitas domiciliares estructuradas por la solicitud. Método: estudio descriptivo, en la modalidad de relato de experiencia, basado en la experiencia de enfermeras en el desarrollo de visitas domiciliaras a partir de la solicitud con 17 mujeres con embarazos de alto riesgo. Resultados: las visitas domiciliaras permitieron a las enfermeras apreciar los aspectos subjetivos del cuidado, comprender las necesidades de la mujer y comprender el embarazo en la vida de las mujeres, aspectos determinantes de las demandas de la atención prenatal. Conclusión e implicaciones para la práctica: la visita domiciliara de enfermeras bajo el auspicio de la solicitud se reveló como una tecnología de atención prenatal de alto riesgo que produce la acogida de necesidades únicas y capaz de conducir la reconstrucción de la atención prenatal en el contexto de embarazo de alto riesgo.

Palabras clave: Atención Prenatal; Enfermeras; Embarazo de Alto Riesgo; Integralidad en Salud; Visita Domiciliaria. 


\section{INTRODUÇÃO}

No Brasil, a Atenção ao Pré-natal de Alto Risco (APNAR) pode vir a ser desenvolvida de forma integral na Atenção Básica (AB) sempre que contar com equipe especializada para esses casos. Em sua falta, ambulatórios especializados estão previstos. De todo modo, para a $A B$, está a coordenação, ordenação e continuidade do cuidado, na direção de garantir direitos e acesso às tecnologias de cuidado em tempo oportuno, com vistas a suprir necessidades singulares. ${ }^{1,2}$

Em 2011, o governo brasileiro instituiu a Rede Cegonha $(\mathrm{RC})$, na prerrogativa de que o cuidado pré-natal seja de risco habitual ou alto risco, assegurasse qualidade de assistência, acesso, articulação das redes e promoção da saúde. Às gestantes, ficou garantido o acolhimento às intercorrências na gestação; avaliação e classificação de risco gestacional; acesso ao prénatal de alto risco em tempo oportuno; realização de exames; e articulação da rede assistencial materno-infantil, na direção de efetivar a integralidade do cuidado. , $^{3,4}$

A APNAR demanda de qualificação no contexto brasileiro, e o fortalecimento dessa atenção está sinalizado como premente para garantir direitos e contrapor aos índices de morbimortalidade materna e neonatal no Brasil. Ao tomar os Objetivos de Desenvolvimento Sustentável, o Brasil assumiu para 2030 redução na taxa de mortalidade materna para 30 mortes por 100 mil nascidos vivos. ${ }^{5}$ Em 2018, a Razão de Mortalidade Materna (RMM) foi de 59,1 óbitos para cada 100 mil nascidos vivos, com predomínio dos óbitos maternos decorrentes de causas obstétricas diretas. ${ }^{5}$

Neste cenário, assinala-se também a relação entre a incidência de morbimortalidade na gravidez de alto risco e a insuficiência de informação dadas à gestante ${ }^{6}$ e família, com desdobramentos deletérios à saúde dela e da criança gestada, complicações na gestação, parto e puerpério, como a mortalidade materna e aumento da mortalidade neonatal. ${ }^{7}$

A assistência em saúde pré-natal centrada e valorizadora do modelo biomédico, tende a ser limitante para o atendimento de outras necessidades de saúde das mulheres que não às morfofuncionais. A prática sob tal paradigma impacta a formação do vínculo entre gestante e profissional, reduz a clínica e acarreta sentimentos de insatisfação e desconfiança pela assistência prestada, inclusive levando às situações de não adesão ao pré-natal. $^{8}$

É premente pensar a assistência às gestantes de alto risco, conectando os emaranhados da produção de cuidado que envolve o social, o técnico, o não técnico, a disponibilidade, o afeto e a empatia. Por isso, pensar o cuidado exclusivamente por meio de dispositivos protocolares limita e torna o pré-natal insuficiente. ${ }^{4}$

Nesse sentido, a visita domiciliar (VD) se destaca enquanto uma alternativa de articulação assistencial possível entre profissionais, gestantes e suas famílias e como prática de cuidado que pode superar as insuficiências. Primeiro, por promover aproximação dos profissionais nos contextos reais de vida dos quais os sujeitos estão imersos, com impacto na produção de cuidado que poucas práticas em saúde têm. ${ }^{9}$ Segundo, pois essa aproximação possibilita a valorização da dimensão subjetiva dos envolvidos nessa relação, construindo espaços de comunicação e diálogo, favorecendo o compartilhamento de práticas e saberes, ${ }^{10}$ vivendo e refletindo sobre a inteireza de todo o processo de gestação.

Em consonância ao parágrafo anterior, uma atitude solícita contribui para uma atitudinal que intenciona a compreensão da totalidade do ser e do processo de gestação na APNAR, adota disponibilidade para relações intersubjetivas e dialógicas, atuando como norteador da produção de cuidado em VDs de modo a transcender o modelo biomédico. Essa atitude é pautada na possibilidade de abertura e acolhimento ao próximo, marcado pelo desejo de "atender da melhor maneira possível a alguma solicitação; com empenho, interesse, atenção", 11:94 e o entendimento é de que tem potencialidade para nortear a presença de enfermeiros em práticas de visitação domiciliar, sobretudo na valorização dos aspectos subjetivos.

Embora a VD seja uma das ferramentas sugeridas pelo Ministério da Saúde (MS) no acompanhamento de gestantes de risco habitual e de alto risco, há carência de conhecimento científico para um número considerável de enfermeiros, visto que muitos compreendem que seja suficiente, em termos de apreensão de necessidades e cuidados, o direcionamento da gestante até a $A B$ ou quando a VD se realiza por intermédio do Agente Comunitário de Saúde (ACS), mesmo que sua prática seja recomendada aos demais profissionais de saúde que integram a rede. ${ }^{12}$

A atenção à gestante de alto risco é inclusa aos serviços especializados, por vezes, uma ruptura da continuidade do cuidado na Atenção Primária à Saúde. ${ }^{1,8}$ No direcionamento da VD no cuidado pré-natal à gestante de alto risco, esta dispõe enquanto estratégia à continuidade do vínculo da equipe de profissionais da Atenção Primária à Saúde, indispensável para a integralidade da assistência à mulher e sua família de forma longitudinal, devendo ser empregada desde o encaminhamento da gestante ao ambulatório de alto risco, perdurando até tempos pós-parto, com alcance às necessidades de saúde demandadas. ${ }^{13}$

Internacionalmente, há inúmeros investimentos em programas de visitação domiciliar (PVD), com a finalidade de promover o cuidado e desenvolvimento materno-infantil. Esses programas priorizam famílias e comunidades de baixa renda e em situações de vulnerabilidade social e econômica enquanto uma alternativa para minimizar vulnerabilidades, mecanismo importante para a equidade. ${ }^{14-16}$

Na mesma direção, o Brasil vem estimulando e implantando programas. O Programa Criança Feliz, desenvolvido pelo Ministério do Desenvolvimento Social, prevê o cuidado com crianças no período da primeira infância por meio de VD e, mais recentemente, publicou um manual de apoio de VDs às gestantes. ${ }^{13}$ Outro PVD, intitulado Programa Jovem Mães Cuidadoras, intenciona o desenvolvimento da parentalidade positiva na primeira infância e na saúde materno-infantil. É ofertado por meio de VDs conduzidas por enfermeiras às mães adolescentes e primigestas. ${ }^{17}$ 
A prática em saúde dialogada tem potencial transformador no equilíbrio entre o subjetivo e o técnico e na construção de projetos terapêuticos que resgatam a autonomia e o empoderamento da mulher. ${ }^{18}$ Assim, no entendimento de que a mulher e a família são os autores de seus cuidados, cabendo ao enfermeiro dar suporte, para que tenham conhecimento e reivindiquem o cuidado que lhe façam sentido, este estudo teve como objetivo relatar a experiência de produção de cuidado no pré-natal de alto risco por meio de VDs estruturadas na solicitude.

\section{MÉTODO}

Trata-se de um relato de experiência sobre a produção de cuidado, por meio de VDs no pré-natal de alto risco desenvolvido por enfermeiras, sob referencial teórico da solicitude de Paul Ricouer. ${ }^{19} \mathrm{O}$ filósofo francês concebe ao termo solicitude o estatuto de benevolência. Muito mais do que uma obrigação moral de respeito, o bem compreende partilha, por vezes assimétrica, entre o dar e o receber, ${ }^{19-21}$ como responsabilidade decorrente do reconhecimento do outro pelo si mesmo e do outro como a si mesmo. ${ }^{20}$

O relato descreve a experiência de duas enfermeiras vinculadas ao programa de pós-graduação strictu sensu que, como parte de atividades de pesquisa e extensão, realizaram VDs às gestantes de alto risco. Este relato é ancorado na intervenção (VD) do projeto intitulado "Alcances de Programa de Visitação no Pré-natal de Alto Risco", desenvolvido em um município do interior do estado de São Paulo, Brasil, cuja atenção pré-natal de alto risco se dá por meio do serviço especializado.

O projeto mencionado seguiu os preceitos ético-legais brasileiros para pesquisas com seres humanos. Foi aprovado por Comitê de ética e está registrado sob o Parecer $n^{\circ} 2.467 .733$ e CAAE 81715317.7.0000.5504.

A visitação domiciliar teve início em agosto de 2018, a partir da captação da primeira gestante de alto risco em acompanhamento no serviço especializado, estendendo-se até março de 2021 com o término das VDs com a última (17aㅡ) gestante participante da pesquisa. As visitas foram prospectadas para ocorrer com frequência quinzenal, desde a captação da gestante até o primeiro mês de vida da(s) criança(s) nascidas. Contudo, à luz do vivenciado em cada visita, as enfermeiras decidiam por inserir, na necessidade, outras visitas.

As gestantes de alto risco que participaram do projeto de pesquisa foram inclusas mediante diagnóstico de gestação de alto risco e deviam possuir idade superior a 18 anos, ou quando adolescente, ser emancipada. Foram excluídas aquelas cujas condições interferiam em habilidades da mulher para se envolver com a visitação, tais como adoecimento mental, severo déficit cognitivo e/ou sensorial.

As reflexões deste relato são referentes às percepções das enfermeiras visitadoras e das enfermeiras de suporte aos alcances em termos de produção de cuidado originada dessa visitação domiciliar. As enfermeiras de suporte são docentes de nível superior que mediavam espaços dialógicos com as enfermeiras visitadoras e despertavam a reflexão sobre a prática desenvolvida nas visitas, clarificando e deliberando novas condutas diante demanda. Esse processo foi de frequência mensal, a partir dos encontros que eram integralmente registrados em atas. Após as discussões dos casos, as enfermeiras visitadoras foram orientadas sobre como proceder e intervir na próxima VD. Como exemplo prático deste suporte, podemos citar o caso do nascimento de uma criança com má formação, em que as enfermeiras de suporte teceram reflexões sobre o acesso, dinâmica das Redes de Atenção e vínculo. Após as reflexões, as enfermeiras visitadoras puderam esclarecer as mulheres acerca de suas necessidades.

\section{RESULTADOS}

Como parte da apresentação dos resultados relativos à produção de cuidado originados, será feita breve contextualização da trajetória de construção e implementação da visitação domiciliar.

A priori, o desenho das VDs estava voltado para a adoção de um protocolo de visitação às gestantes de alto risco e, inclusive, três visitas foram realizadas sob esta condução. Contudo, buscava-se uma prática de cuidado que promovesse encontros genuínos entre enfermeira visitadora e gestante de alto risco, que direcionasse para a compreensão da totalidade do fenômeno e para as reais necessidades das mulheres e família. Deste modo, a percepção das enfermeiras da equipe foi de que seguir o protocolo trazia rigidez e engessava o encontro, destituía sua natureza dialogada e pautada na corresponsabilização e construção colaborativa do cuidado. O protocolo impedia a apresentação das necessidades pela mulher e família, tendendo a impor pautas que não eram as clamadas pela mulher.

Assim, diante das inquietações sobre a possibilidade de edificar cuidado singular diante da adoção de um guia protocolar e o que fazer com as pautas ali ponderadas - situação de risco gestacional, questões referentes ao parto e nascimento, direitos, família e aleitamento materno - foi tomada a decisão de apostar nas interações vivenciadas e nas subjetividades de cada ser que se desvela em cada oportunidade de interação. Neste sentido, o modelo protocolar foi deslocado para um lugar secundário a ser movimentado na dependência do exposto por cada mulher

Passou-se, então, a adotar uma prática de cuidado por meio de VDs que encorajassem as gestantes a participarem da produção de cuidado, quando foi deliberadamente incorporado o referencial teórico da solicitude de Paul Ricouer ${ }^{19}$ aos modos como andaríamos na VD. O entendimento foi de que ele favorecia uma atitudinal profissional que se direcionava para compreensão da totalidade do fenômeno e requeria disponibilidade para relações intersubjetivas e diálogo, aspecto que foi sendo confirmado ao longo das visitas.

\section{A VISITA DOMICILIAR ENQUANTO PRÁTICA DE CUIDADO}

Ao todo, 17 mulheres gestantes de alto risco aceitaram e receberam VDs, desde o diagnóstico até o primeiro mês de vida da(s) crianças(s) nascida(s). Um total de 130 visitas 
foram realizadas, com tempo médio de uma hora por visita. Elas eram consultadas pelos profissionais do ambulatório de acompanhamento de gestantes de alto risco acerca do desejo de integrar a intervenção e, na confirmação de interesse, eram apresentadas às enfermeiras visitadoras.

Na primeira VD às gestantes, as enfermeiras visitadoras disparavam o encontro por meio das colocações "Conte-me sobre você" e "Conte-me sobre sua vida e gestação" como incentivo para que fizessem uma apresentação de si e de suas vidas, dando a elas a liberdade e um espaço ativo de fala. Fato que se mostrou relevante e potente para promover a compreensão do fenômeno e um encontro consigo, uma vez que os espaços oportunizados para isso no pré-natal habitual eram praticamente nulos, já que, pelo relato delas, imperava um olhar centrado no desenvolvimento da gestação. As mulheres se queixavam e denunciavam este modo de ser apreendidas, assim, póstero as VDs, os relatos eram discutidos com as enfermeiras de suporte para reflexão e direcionamento das intervenções.

As visitas subsequentes estiveram pautadas na aposta na interação, seguindo os movimentos de cada mulher, as necessidades trazidas por elas. No entanto, sob o entendimento de que estávamos também a exercer um importante papel assistencial, foram abordadas temáticas do contexto-materno infantil, sempre atrelado ao exposto pela mulher e suas revelações, para construção de sentidos. O planejamento e a abordagem das temáticas foram prospectados de forma que transcendessem a ordem biomédica e não edificassem as interações que estavam a ser construídas.

A construção de interações pautadas no diálogo, na reciprocidade, na compaixão e na disponibilidade das enfermeiras visitadoras em estar com as gestantes recebeu influência direta do referencial teórico. Para o desenvolvimento das visitas, houve compreensão de que a relação com o outro se dá de forma espontânea, posto que há reconhecimento do outro pelo si mesmo e do outro como a si mesmo. ${ }^{20} \mathrm{O}$ contexto da solicitude reclama exigências que não são inatas ao indivíduo, exigências essas que, portanto, puderam ser apreendidas enquanto a VD foi se estabelecendo como uma prática de cuidado.

Reflexo disso, à medida que as VDs iam acontecendo, as gestantes foram compreendendo o domicílio como uma possibilidade de espaço de cuidado. Encorajadas a dialogar, foram desenvolvendo confiança, vínculo com as enfermeiras visitadoras, sentindo-se livres para a troca e o diálogo. Revelavam a si de forma autêntica, participando da produção de cuidado, ora compartilhando suas dúvidas, ora levantando temáticas a serem exploradas e assim, sucessivamente, nas outras visitas. Sobretudo no quesito tempo, as gestantes oportunizaram o momento do cuidado em domićlio, visto que o espaço do consultório médico não explorava a participação delas, outra queixa recorrente.

Um ponto a ser destacado na produção do cuidado é que as enfermeiras visitadoras utilizaram da estratégia do cuidado longitudinal. Em outras palavras, foi acordado manter a mesma enfermeira visitadora desde o início das visitas até o fim de seu desenvolvimento, no intuito de fortalecer a vinculação e promover uma interação mais efetiva, aspecto esse que foi alcançado com sucesso na produção do cuidado. Esse aspecto também favoreceu a consolidação do vínculo entre enfermeira e gestante, a confiança, a segurança e a autenticidade na interação.

Por fim, sob o ponto de vista de todas as enfermeiras envolvidas com a experiência, esta promoveu crescimento profissional e pessoal. Como ponto em comum, antes da experiência, já acreditávamos na possibilidade de produções de cuidado a partir de encontros genuínos. Após, saímos ainda mais fortalecidas para estimular o cuidado e práticas que valorem o respeito, a solicitude e, que sejam, sobretudo, centradas nas necessidades reais de saúde e no protagonismo da mulher. Nesta direção, é essencial alcançar disponibilidade e encontro autêntico, os quais têm nos quesitos atitude solícita do enfermeiro e em sua ida ao domicílio determinantes profícuos e promotores do encontro autêntico, promotor moral do acolhimento de necessidades, com produção de cuidado singular.

\section{DISCUSSÃO}

A experiência relatada permitiu refletir sobre a potencialidade da VD sob o referencial da solicitude como promotora e edificadora de cuidado singular e integral.

A prática da visita por si só, sem uma ancoragem para o atitudinal do profissional, não é suficiente para alcances dialógicos e reveladores das necessidades. A partir da perspectiva da solicitude, do entendimento das gestantes como protagonistas e da valorização das interações a cada encontro, a VD da forma como foi aqui relatada permitiu o envolvimento relacional, fortalecimento do vínculo e favoreceu a produção do cuidado.

O ponto de partida para reconstrução do cuidado às gestantes de alto risco nesta experiência é a consideração de que as relações de cuidado que são construídas no serviço especializado, ou até mesmo na $A B$, situam-se exclusivamente no fenômeno do diagnóstico gestacional, no iminente risco de morbi e mortalidade materna e infantil. ${ }^{22}$ Observa-se que os espaços forjados para construir o cuidado precarizam a interação dos envolvidos e a renegam a segundo plano, pois olham os processos relacionais e os encontros produzidos pelos sujeitos como obscurantismo, em detrimento à valorização de outros saberes, ditos mais científicos. ${ }^{23,24}$

Assim, a produção de cuidado implica encontros. ${ }^{23}$ Para o desenvolvimento de uma relação que adote um paradigma de reconstrução, é necessário a inclusão efetiva e ativa do usuário e, no presente caso, a gestante de alto risco enquanto protagonista de sua própria vida, inclusive nas circunstâncias de fragilidade emocional e biológica, ${ }^{25}$ como o fenômeno do viver o alto risco remete.

Faz-se necessário descontruir o lugar em que, hegemonicamente, as gestantes e as mulheres, em geral, foram sendo colocadas, como objeto das ações e práticas de saúde. Só assim e, a partir daí, a produção do cuidado será motivada na perspectiva de um modelo mais integral. 23,25 
Soma-se à $A B$ sua organização em territórios delimitados e por ter a VD em seu conjunto de práxis. ${ }^{26}$ Posta na Política Nacional de Atenção Básica (PNAB), desde 2017, foi recomendada à $A B$ uma prática assistencial ao encontro do Cuidado Centrado na Pessoa. Conforme Moira, ${ }^{27}$ esse cuidado se dá quando os protagonistas encontram um campo comum para abordar preocupações e, então, são viabilizadas trocas para o poder decisório das questões de saúde. Para tanto, esperam-se dos profissionais de saúde atitudes que valorem a autonomia e o protagonismo da pessoa, assim como do próprio profissional enquanto pessoa.

A VD, nesse sentido, pode ser trabalhada e utilizada como uma alternativa e ponto de partida de novas interações com gestantes de alto risco. O espaço do domicílio promoveu maior segurança, conforto e autonomia às mulheres na produção do cuidado. Sobretudo, no reconhecimento como espaço que remete às lembranças da gestação, o viver e o estar agora, ${ }^{23}$ fez com que as mulheres se expressassem livremente junto às enfermeiras visitadoras, compartilhando sua vida, suas angústias e felicidades, reconhecendo o lugar de destaque de cada uma no viver o alto risco.

Nesse ínterim, para que este reconhecimento de si seja legítimo e contínuo, infere-se aqui que os PVD, internacionalmente difundidos, devem reforçar a informação de como ocorre a produção de cuidado por meio das visitas. No entendimento de que gestantes de alto risco, sobretudo as mulheres em situações de vulnerabilidade e pobreza, necessitam e possuem desfechos mais positivos quando recebem VDs, ${ }^{16,27}$ tal cuidado deve ser difundido pelos profissionais de saúde, visando os inúmeros benefícios para a mulher no período de gestação, ${ }^{28}$ bem como a janela de oportunidade do período gestacional para outras questões da vida, a exemplo de enfrentamento de violências, parentalidade, questões de saúde mental, entre outras.

Por fim, cabe ressaltar que a produção de cuidado, mesmo que por meio das VDs, espaço privilegiado para a construção de relações, implica às enfermeiras ou a outros profissionais de saúde atitudes que superem a unilateralidade dos paradigmas biomédicos, com investimento nas relações dialógicas e ampliação do olhar e da escuta para a complexidade da vida da gestante. ${ }^{29}$

Por acreditar que esse atitudinal pode ser desenvolvido enquanto se cuida, o conceito de solicitude se fez pertinente na experiência da condução de VDs no presente estudo. A solicitude compreende que nem todas as relações intersubjetivas se dão entre iguais. Em outras palavras, contempla relações com pessoas oprimidas em situação de vulnerabilidade e sofrimento, por quaisquer que sejam os motivos. Assim, partindo de tal pressuposto, faz-se necessário compreender e aceitar as diferenças existentes, respeitando o outro para além das questões morais. O conceito de solicitude é pautado na possibilidade de abertura e acolhimento ao próximo e devolve o equilíbrio de reciprocidade entre as relações desiguais. ${ }^{21}$

Para Ricouer, ${ }^{19}$ a proposta da solicitude não deve ser confundida com piedade, pois todas as relações envolvem reciprocidade, ainda que a pessoa que está a receber o cuidado só compartilhe o seu sofrimento para com o outro que está a compartilhar compaixão e simpatia

Trazendo para a presente experiência, a produção envolveu encontros entre gestantes e enfermeiras na prática da VD. Os resultados revelados e anteriormente prospectados só floresceram diante do ambiente terapêutico favorável que se criou. A enfermagem e a VD já possuem potencialidade para tal concepção, ${ }^{22}$ mas só se concretizou em meio à solicitude.

A escolha do enfermeiro enquanto profissional visitador foi intencional, quando se pensou que, para além do conhecimento técnico-científico, traz em sua formação acadêmica alicerces da humanização que favorecem o acolhimento e criação de vínculo. Destarte, o diferencial do enfermeiro é construído através do cuidado, quando aberto ao devir, revela-se e externa seus saberes e suas intencionalidades, processo esse que se dá através da interação humana no reconhecimento do enfermeiro enquanto um ser de relação. ${ }^{30}$

\section{CONSIDERAÇÕES FINAIS E IMPLICAÇÕES PARA A PRÁTICA}

A experiência de desenvolver e executar VDs enquanto prática de cuidado e sob os auspícios da solicitude demonstrou ser ferramenta que promove a produção de cuidado através das relações estabelecidas. Na ótica das autoras, a experiência potencializou ainda mais o entendimento de que as interações intersubjetivas são necessárias para o cuidado e se revelam a partir dele. Para as gestantes que receberam VDs, pressupõese que a vivência de produção do cuidado foi transformadora e significativa no viver a gravidez sob o alto risco gestacional.

Considerando que o fenômeno de gestar em alto risco envolve diversos aspectos, parece-nos que a produção de cuidado aqui exposta é inconclusiva. Assim, enquanto limites, percebe-se que há outros aspectos que podem ser buscados e fortalecidos na visitação domiciliar, a exemplo da perspectiva de direitos e a produção de cuidado com outros sujeitos que envolvam a vida da gestante de alto risco, a exemplo do companheiro, da família e do próprio emaranhado de rede de serviços utilizados por essas gestantes. Assim, no intuito de que esse trabalho não se finde aqui, sugerimos outras pesquisas com visitação domiciliar às gestantes de alto risco.

Elementos, como o acesso, a equidade, a vulnerabilidade e a integralidade em saúde se fizeram presentes na presente experiência, possibilitando a construção de um cuidado que superasse as insuficiências do olhar exclusivamente técnico e biomédico usual na atenção em saúde brasileira. A VD traduziu e acolheu as reais necessidades de saúde das gestantes, traspondo o risco gestacional, mas também frente às condições de vida e funcionamento familiar, quando enfermeiras se disponibilizaram verdadeiramente à gestante, a lutar com elas por acolhimento de necessidades e direito negados.

Esperamos que esta experiência sensibilize os profissionais de saúde, sobretudo os enfermeiros, na produção de cuidados 
e que busquem reconstruir as práticas no fenômeno do gestar em alto risco, assim como nos tocou e nos motivou para tal.

\section{CONTRIBUIÇÕES DOS AUTORES}

Desenho do relato de experiência. Bruna Felisberto de Souza. Monika Wernet.

Levantamento de informações. Bruna Felisberto de Souza. Bruna de Souza Lima Marski.

Análise da experiência. Bruna Felisberto de Souza. Bruna de Souza Lima Marski. Maria Aparecida Bonelli. Mariana Torreglosa Ruiz. Monika Wernet

Interpretação. Bruna Felisberto de Souza. Bruna de Souza Lima Marski. Maria Aparecida Bonelli. Mariana Torreglosa Ruiz. Monika Wernet

Redação e revisão crítica do manuscrito. Bruna Felisberto de Souza. Bruna de Souza Lima Marski. Maria Aparecida Bonelli. Mariana Torreglosa Ruiz. Monika Wernet

Aprovação da versão final do artigo. Bruna Felisberto de Souza. Bruna de Souza Lima Marski. Maria Aparecida Bonelli. Mariana Torreglosa Ruiz. Monika Wernet

Responsabilidade por todos os aspectos do conteúdo e a integridade do artigo publicado. Bruna Felisberto de Souza. Bruna de Souza Lima Marski. Maria Aparecida Bonelli. Mariana Torreglosa Ruiz. Monika Wernet.

\section{EDITOR ASSOCIADO}

Stela Maris de Melo Padoin ()

\section{EDITOR CIENTÍFICO}

Ivone Evangelista Cabral ๑)

\section{REFERÊNCIAS}

1. Portaria $\mathrm{n}^{\circ} \mathbf{1 0 2 0}$, de 29 de maio de 2013 (BR). Institui as diretrizes para a organização da Atenção à Saúde na Gestação de Alto risco e define os critérios para a implantação e habilitação dos serviços de referência à Atenção à Saúde na Gestação de Alto Risco, incluída a Casa da Gestante, Bebê e Puérpera (CGBP), em conformidade com a Rede Cegonha. Diário Oficial da União, Brasília (DF), 29 maio 2013.

2. Miranda EF, Silva AMN, Mandú ENT. Approaching health needs by nurse in prenatal consultation. R Pesq Cuid Fundam Online. 2018;10(2):52433. http://dx.doi.org/10.9789/2175-5361.2018.v10i2.524-533.

3. Ministério da Saúde (BR), Secretaria de Atenção à Saúde. Manual prático para implementação da Rede Cegonha. Brasília (DF): Ministério da Saúde; 2011.

4. Carvalho MMR, Jorge MSB, Franco TB. "My child gave me my life back": cartography of the "Rede Cegonha". Interface. 2018;22(66):757-67. http://dx.doi.org/10.1590/1807-57622016.0503.

5. Ministério da Saúde (BR). Brasil reduziu $8,4 \%$ a razão de mortalidade materna e investe em ações com foco na saúde da mulher [Internet]. 2020 [citado 2021 ago 2]. Disponível em: https://www.saude.gov.br/ noticias/agencia-saude/46970-brasil-reduziu-8-4-a-razaode-mortalidadematerna-e-investe-em-acoes-com-foco-na-saude-da-mulher

6. Pedrini L, Prefumo F, Frusca AV, Ghilardi A. Counselling about the risk of preterm delivery: a systematic review. BioMed Res Int. 2017;2017:7320583. http://dx.doi.org/10.1155/2017/7320583. PMid:28848765.

7. Andrade FM, Castro JFL, Silva AV. Percepções das gestantes sobre as consultas médicas e de enfermagem no pré-natal de baixo risco.
Enf Cent O Min. 2016;6(3):2377-88. https://doi.org/10.19175/recom. v6i3.1015

8. Ferreira SN, Lemos MP, Santos WJ. Representações sociais de gestantes que frequentam serviço especializado em gestações de alto risco. Rev Enferm Cent-Oeste Min. 2020;10:e3625. http://dx.doi.org/10.19175/ recom.v10i0.3625.

9. Seixas CT, Merhy EE, Feuerwerker LCM, Santo TBE, Slomp H Jr, Cruz KT. Crisis as potentiality: proximity care and the epidemic by COVID-19. Interface. 2021;25(Supl. 1):e200379. http://dx.doi.org/10.1590/ interface.200379.

10. Paim JS, Almeida N Fo. Saúde coletiva: uma "nova saúde pública" ou campo aberto a novos paradigmas? Rev Saude Publica. 1998;32(4):299316. http://dx.doi.org/10.1590/S0034-89101998000400001.

11. Otero MF. Alteridade e solicitude a partir da obra o si mesmo como outro de Paul Ricouer. Rev Enciclopédia. 2015;4(1):94-101.

12. Medeiros LS, Costa ACM. Período puerperal: a importância da visita domiciliar para enfermeiros da Atenção Primária à Saúde. Rev Rene. 2016;17(1):112-9. http://dx.doi.org/10.15253/2175-6783.2016000100015.

13. Ministério da Cidadania (BR). Visitas domiciliares às gestantes. Brasília (DF): Ministério da Cidadania; 2020.

14. Olds DL. Prenatal and infancy Home Visiting by nurses: from randomized trials to community replication. Prev Sci. 2002;3(3):153-72. http://dx.doi. org/10.1023/A:1019990432161.

15. Olds DL, Robinson J, Pettitt L, Luckey DW, Holmberg J, Ng RK et al. Effects of home visits by paraprofessionals and by nurses: age 4 followup results of a randomized trial. Pediatrics. $2004 \mathrm{Dec} 01 ; 114(6): 1560-8$. http://dx.doi.org/10.1542/peds.2004-0961.

16. Radcliff E, Breneman CB, Crouch E, Baldwin I. Are we serving the most at-risk communities? Examining the reach of a South Carolina Home Visiting Program. J Community Health. 2019;44(4):764-71. http://dx.doi. org/10.1007/s10900-018-00606-5.

17. Fracolli LA, Reticena KO, Abreu FCP, Chiesa AM. The implementation of a home visits program focused on parenting: an experience report. Rev Esc Enferm USP. 2018;52:e03361.

18. Campagnoli M, Silva CP, Resende RCP. Atendimento de pré-natal na estratégia saúde da família: singularidade da assistência de enfermagem. Rev Nursing. 2019;22(251):2915-20. http://dx.doi.org/10.36489/ nursing.2019v22i251p2915-2920.

19. Ricouer P. O si mesmo como um outro. Tradução de Lucy Moreira Cesar. Campinas: Papirus; 1991.

20. Martini RMF. Paul Ricoeur: a ética da solicitude questiona a educação Pro-Posições. 2016;27(2):235-52. http://dx.doi.org/10.1590/1980-62482015-0016.

21. Rossatto ND. O respeito ao outro na ética de Paul Ricouer. Dissertatio. 2018:8:125-39. https://doi.org/10.15210/dissertatio.v0io.14585.

22. Errico LSP, Bicalho PG, Oliveira TCFL, Martins EF. The work of nurses in high-risk prenatal care from the perspective of basic human needs. Rev Bras Enferm. 2018;71(Supl.3):1257-64. http://dx.doi.org/10.1590/00347167-2017-0328. PMid:29972522.

23. Feuerwerker LCM. Cuidar em saúde. In: Bertussi DC, Merhy EE, organizadores. Avaliação compartilhada do cuidado em saúde surpreendendo o instituído nas redes. Rio de Janeiro: Ed. Hexis; 2016. p. 35-47.

24. Azeredo YN, Scharaiber LB. Authority, power and violence: a study on humanization in health. Interface. 2021;25:e190838. http://dx.doi. org/10.1590/interface.190838.

25. Fernandes JA, Campos GWS, Francisco PMSB. Perfil das gestantes de alto risco e a cogestão da decisão sobre a via de parto entre médico e gestante. Saúde Debate. 2019;43(121):406-16. http://dx.doi. org/10.1590/0103-1104201912109.

26. Flor CR, Oliveira CDL, Cardoso CS, Rabelo CF, Gontijo BL, Carvalho SF et al. Primary health care as assessed by health professionals: comparison of the tradicional model versus the Family Health Strategy. Rev Bras Epidemiol. 2017;20(4):714-26. http://dx.doi.org/10.1590/19805497201700040013. 
27. Moira S. Medicina centrada na pessoa: transformando o método clínico. Tradução de Anelise Burmeister, Sandra Maria Mallmann da Rosa. Porto Alegre: Artmed, 2017.

28. Barkin JL, Bloch JR, Smith KER, Telliard SN, McGreal A, Sikes C et al. Knowledge of and attitudes toward perinatal home visiting in women with high-risk pregnancies. J Midwifery Womens Health. 2021 Mar/ Apr;66(2):227-32. http://dx.doi.org/10.1111/jmwh.13204.
29. Amorim TV, Souza ÍEO, Salimena AMO, Queiroz ABA, Moura MAV, Melo MCSC. Reproductive risk in pregnant women with heart diseases: the living world guiding health care. Texto Contexto Enferm. 2018;27(2):e3860016. http://dx.doi.org/10.1590/0104-070720180003860016.

30. Ramírez-Pérez M, Cárdenas-Jiménez M, Rodríguez-Jiménez S. El Dasein de los cuidados desde la fenomenología hermenéutica de Martín Heidegger. Enferm Univ. 2015;12(3):144-51. http://dx. doi.org/10.1016/j. reu.2015.07.003.

\footnotetext{
a Artigo extraído de tese de doutorado em andamento "Alcances de visitação domiciliar no pré-natal de alto risco", autoria de Bruna Felisberto de Souza Programa de Pós-Graduação em Enfermagem, Universidade Federal de São Carlos, 2021.
} 